\title{
Performance measurement of small-cells deployed under a heterogeneous network: an analysis of the co-existence of small-cells with the macro囚cell in 5G NR
}

Mobasshir Mahbub ( $\square$ mbsrmhb@gmail.com)

Ahsanullah University of Science and Technology https://orcid.org/0000-0002-8272-7208

\section{Bobby Barua}

Ahsanullah University of Science and Technology

\section{Research Article}

Keywords: 5G, Small-cell, Macro-cell, HetNet

Posted Date: July 19th, 2021

DOl: https://doi.org/10.21203/rs.3.rs-372229/v1

License: (c) (1) This work is licensed under a Creative Commons Attribution 4.0 International License.

Read Full License 


\title{
Performance measurement of small-cells deployed under a heterogeneous network: an analysis of the co-existence of small-cells with the macro-cell in 5G NR
}

\author{
Mobasshir Mahbub* \& Bobby Barua \\ Department of Electrical and Electronic Engineering, Ahsanullah University of Science and Technology, Dhaka, Bangladesh \\ *Email: mbsrmhb@gmail.com
}

\begin{abstract}
Advancements of cellular networks such as $4 \mathrm{G}$ and $5 \mathrm{G}$ proposed the collaboration of small-cell technologies in mobile networks and constructed a heterogeneous network (HetNet) for collaborative connectivity. There are many benefits of small-cell-based collective communication such as the increase of device capability in indoor/outdoor locations, enhancement of wireless coverage, improved signal efficiency, lower implementation costs of gNB (Next-generation Base Station introduced in 5G), etc. The integration of small-cells by deploying low-power BSs (base stations) in conventional macrogNBs was investigated as a convenient and economical way of raising the potentials of a cellular network with high demand from consumers. The fusion of small-cells with macro-cells offers increased coverage and capacity for heterogeneous networks. Therefore, the research aimed to realize the performance of a small-cell deployed under a macro-cell in a two-tier heterogeneous network. The research first modified the reference equation for measuring the received power by introducing the transmitter and receiver gain. The paper then measured the SINR, throughput, spectral efficiency, and power efficiency for both downlink and uplink by empirical simulation. The research further enlisted the notable outcomes after examining the simulation results and discussed some relevant research scopes in the concluding sections of the paper.
\end{abstract}

Keywords - 5G, Small-cell, Macro-cell, HetNet.

\section{Introduction}

Propelled by the explosion of increasingly evolving gadgets and the introduction of new services, the mobile and wireless network traffic is increasing geometrically each year and this increment is continuing persistently. From 2010 to 2025, a 1000times rise in data traffic is predicted with a further 10 to 100times rise in 2020 to 2030 , as per projection and statistical research conducted by the International Telecommunications Union (ITU) [1], [2]. Several advanced technologies are needed to counter this rapid increase in data traffic to satisfy constantly growing data offloading traffic and network demand. As a result of the transition of the cellular network to LTE and 5G NR, the network has emerged as a multi-tier or multi-level network that incorporates a traditional mobile network with many lowpowered base stations (e.g., small-cells). Large-scale deployment of small-cells in such HetNets, including pico-cells and femto-cells underlaid inside a macro-cell network, is indeed one of the promising strategies for addressing the growing wireless traffic [3].

According to the statistics, approximately $67 \%$ of the cellular operators have already deployed indoor small-cells (femto-cells), and the deployment of all types of small-cell (e.g. pico-cells, femto-cells) increased from 4.3 to 36.8 million in the year 2015 . More than 40,000 outdoor small-cells have already been installed by the end of 2016 [4], [5]. A small-cell BS (SBS) is a cost-effective, low-power radio transmission system built to cover a short coverage region. It can be implemented in plugand-play mode, having the capability of self-configurations according to the required transmission parameters and does not require sophisticated maintenance [6], [7].

Moreover, small-cell networks reflect a change of approach from conventional central macro-cell BSs to a more self-organized model in which small-cells are used in all considerable environments, including both indoors and outdoors, in combination with existing macro-cells. However, a new set of critical architectural problems are emerging from the coexistence of various types of network equipment with different requirements on the same scale. These primary issues must immediately be tackled so that the promised advantages of small-cell solutions are completely achieved [8].

Small-cells have shorter coverage areas and significantly less transmission power to provide network services to their associated UEs with greater service efficiency (QoS) and higher data rates. Nevertheless, the spontaneous deployment of a dynamic collection of small-cells within HetNets complicates resource management techniques; because multiple tiers of small-cell base stations, e.g., the femto-tier and pico-tier, respectively, have distinct transmission characteristics. The macro-cell base stations (MBSs) are usually deployed to offer wide coverage and broadband connectivity to the user equipment (UE) through high power transmission [9], [10]. Comparatively, within a shorter range, pico-cells operate with a licensed radios spectrum having medium power of transmission power and offload data traffic from the macro-cell in hotspot coverage areas.

To ensure efficient performance of small-cells deployed under a heterogeneous network (with respect to allocated network resources) prior measurements and analyses considering several network performance criteria are necessary.

The paper presented research that represents the SINR, throughput, spectral efficiency, and power efficiency 
performance of a small-cell (outdoor) deployed under a macrocell.

The interesting thing is that the research performed in this paper is based on such formulas that are unique in terms of the performance measurement of small-cell (the formulas previously utilized to analyze communication link rather than small-cell or HetNet). Fig. 1 shows the considered two-tier heterogeneous network formed by seven small-cells operating under one macro-cell.
In the following sections of this paper, section 2 describes the related works, literature, and contributions relevant to this work. Section 3 includes models for measurements (equations/formulas). Section 4 included the simulation results with brief discussions. Section 5 includes notable outcomes of the research and future research directions. Section 6 is the concluding section for this article.

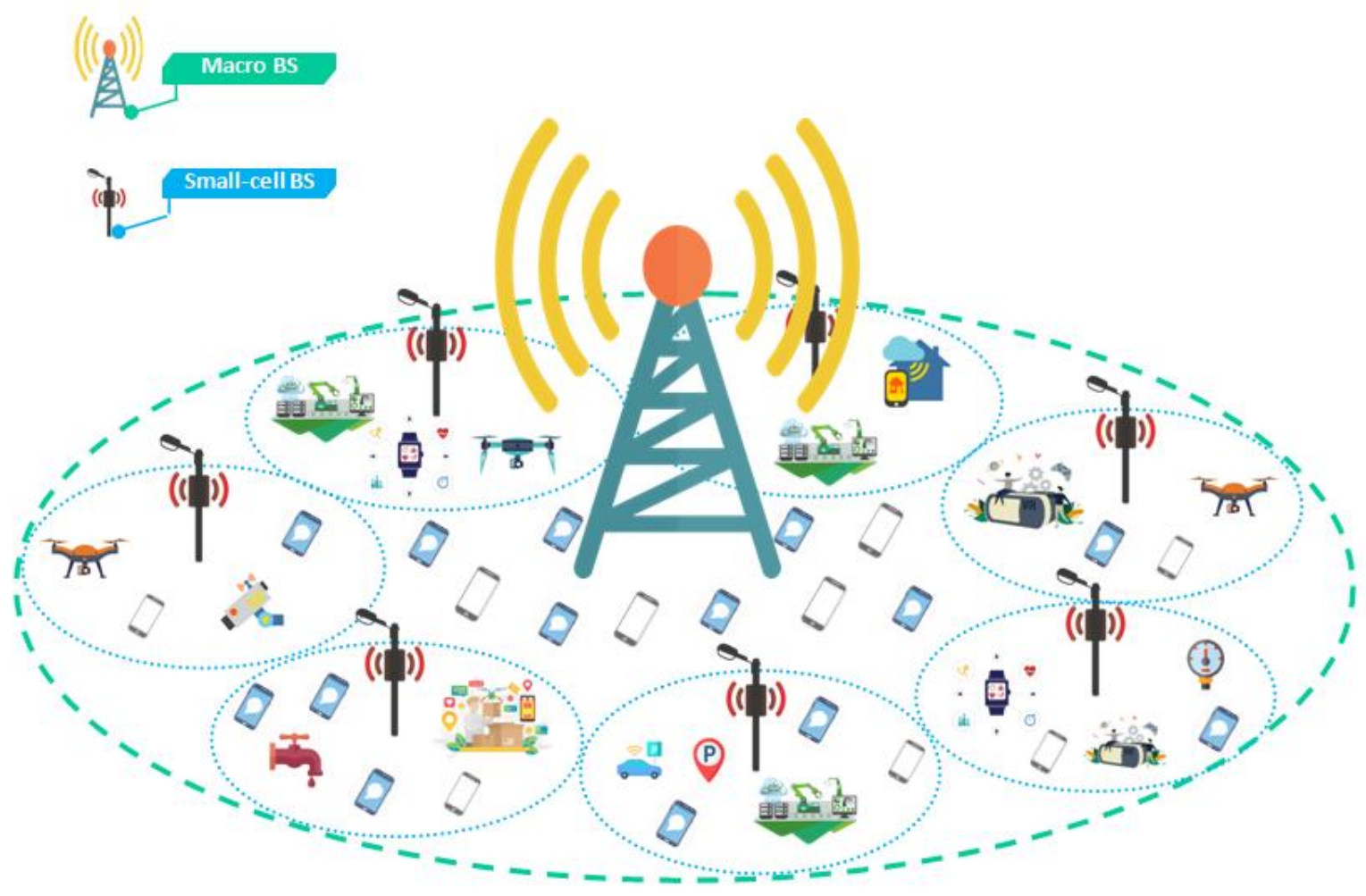

Fig. 1: SBSs deployed under an MBS in a two-tier HetNet

\section{Related Works and Contributions}

In this section, the paper has analyzed several pieces of research previously performed on the deployment of small-cell in a heterogeneous network. It mainly focused on the performance of small-cell deployed under the macro-cell with respect to interference management (from macro-cell and small-cell tier), corresponding throughput, and spectral efficiency.

The research of Ramos et al. [11] explores the average SINR and the throughput of 5G Small-cell HetNet operating at 2.6, 3.5, and $5.62 \mathrm{GHz}$. Elkourdi et al. [12] considered network throughput as the prime performance parameter to figure out the optimal placement locations for small-cells. Wu et al. [13] in this paper analyzed the deployment of small-cells to meet the data rate requirement of $5 \mathrm{G} \mathrm{NR}$ utilizing stochastic geometry. A twotier Hetero-cellular network (HCN) which consists of macrocells and small-cells is utilized as the network model. Shimodaira et al. [14] also considered network throughput as the prime performance parameter for the deployment of small-cells. Pratap et al. [15] studied the resource allocation approach for spectrum reuse maximization, minimization of interference, and user-level fairness in small-cells for heterogeneous $5 \mathrm{G}$ networks. Pak et al. [16] analyzed effective deployment of small-cell with respect to SINR performance. Haija et al. [17] investigated the impact of cooperation of small and macro-cell (SMC) for enhancing the spectral efficiency and network reliability in the case of uplink in a HetNet. Guo et al. [18] proposed a framework to maximize the efficiency (spectral) of the cellular network and to minimize interference generated by the deployment of smallcell. Maltsev et al. [19] considered a cellular network with a few millimeter-wave (mmWave) small-cells under LTE macro-cells. The influence of interactive interference in the mmWave band (57 to $64 \mathrm{GHz}$ ) is evaluated. Kazmi et al. [20] analyzed resource allocation to maximize the network throughput of small-cells under the cross-tier interference constraint. Pervaiz et al. [21] have investigated the trade-off between spectral efficiency and 
energy efficiency in D2D-enabled uplink transmission in the case of heterogeneous networks.

Contribution of this Research: The research has reviewed the aforementioned papers (closely relevant to this research) to gather knowledge about prior works and to find out research gaps. The research interestingly found that most of the article had performed measurements on either uplink or downlink. The notable matter regarding this research is that it has considered both uplink and downlink. Another important point is that the research has modified the reference formula by including transmitter-receiver gain. Moreover, the research has performed measurements considering the SINR, throughput, spectral efficiency, and power efficiency, and all of these measurements altogether in a single article were absent earlier according to the authors' best expertise. These all might be considered as the notable extensions introduced by the research.

\section{System Model}

The research considered a two-tier heterogeneous network (HetNet) where small-cells are laid within the macro-cell network. Considering a geo-location $\mathfrak{D} \subset \mathfrak{R}^{2}$ in which wireless user equipment (UE) are situated in a two-dimensional plane. A set $\boldsymbol{M}$ of $M$ small-cell base stations (SBS) are deployed as terrestrial base stations to serve corresponding UEs. The network traffic in the heterogeneous network is presumed as a full buffer. Contemplating an SBS $i \in \mathfrak{M}$ is serving its corresponding UEs $u$ $\in i$. Let, $P_{i}^{S C B S}$ and $B_{i}^{S C B S}$ are maximum transmit power and bandwidth per-channel designated to serve the UE. Similarly, $P_{u \in \mathrm{i}}^{U E}$ and $B_{u \in \mathrm{i}}^{U E}$ are transmission power and bandwidth of UE. $P^{M B S}$ denotes the maximum transmission power of a macro-cell BS [22].

Thereby the received power in terms of downlink (SBS-to-UE) can be calculated using the following formula (Eq. 1),

$$
\bar{P}_{r, u \in i}^{U E}=\frac{P_{i}^{S c B S} G_{t} G_{r} \delta_{t, r}}{D_{t, r}^{\alpha}}
$$

where $G_{t}$ and $G_{r}$ represent the transmitter and receiver antenna gain. $\delta_{t, r}$ denotes the Rayleigh fading coefficient that follows independent exponential distributions with unit mean e.g. $\delta_{t, r^{\sim}}$ $\exp (1) . \alpha$ is the path loss exponent whose value is usually $\geq 2$. $D_{t, r}$ is the transmitter-receiver separation distance in meters. Similarly, the received power in the uplink (UE-to-SBS) can be measured by (Eq. 2),

$$
\bar{P}_{r, \mathrm{i}}^{S c B S}=\frac{P_{u \in \mathrm{i}}^{U E} G_{t} G_{r} \delta_{t, r}}{D_{t, r}^{\alpha}}
$$

Since the received power for the downlink and uplink is derived the SINR can be calculated. The downlink SINR can be measured using the following equation (Eq. 3),

$$
\zeta_{u \in i}^{U E}=\frac{\bar{P}_{r, u \in i}^{U E}}{I_{i}+I_{M B S}+N}
$$

where $I_{i}=\beta \sum_{j \neq i}^{n} \bar{P}_{r, u \in i}^{U E}$ denotes the interference received by the UE from other SBSs of the same tier rather than serving SBS $i$. $\beta$ is the adjustment factor or weight-factor valued among 0 to 1. $I_{M B S}=\frac{P^{M B S} G_{t} G_{r} \delta_{t, r}}{D_{t, r}^{\alpha}}$ denotes the interference received by the UE from the MBS. $P^{M B S}$ denotes the transmission power of MBS. $N=-174+\operatorname{lolog}_{10}($ Bandwidth in $\mathrm{Hz}$ ) denotes the noise power.

Similarly, the uplink SINR can be measured by (Eq. 4),

$$
\zeta_{i}^{S c B S}=\frac{\bar{P}_{r, \mathrm{i}}^{S c B S}}{I_{i}+I_{M B S}+N}
$$

Thereby the throughput in terms of the downlink is measured by the following formula (Eq. 5),

$$
\bar{C}_{u \in i}^{U E}=B_{i}^{S c B S} \log _{2}\left(1+\zeta_{u \in i}^{U E}\right)
$$

The uplink throughput can be measured by (Eq. 6),

$$
\widetilde{C}_{i}^{S c B S}=B_{u \in i}^{U E} \log _{2}\left(1+\zeta_{i}^{S c B S}\right)
$$

The spectral efficiency for downlink can be calculated by (Eq. 7),

$$
\eta_{u \in i}^{U E}=\log _{2}\left(1+\zeta_{u \in i}^{U E}\right)
$$

The uplink spectral efficiency thereby measured by (Eq. 8),

$$
\eta_{i}^{S c B S}=\log _{2}\left(1+\zeta_{i}^{S c B S}\right)
$$

The power efficiency for downlink can be calculated using the following formula (Eq. 9),

$$
\mathcal{E}_{u \in i}^{U E}=\frac{B_{i}^{S c B S} \log _{2}\left(1+\zeta_{u \in i}^{U E}\right)}{P_{i}^{S c B S}}
$$

The power efficiency in terms of uplink can be measured by (Eq. $10)$,

$$
\varepsilon_{i}^{S c B S}=\frac{B_{u \in i}^{U E} \log _{2}\left(1+\zeta_{i}^{S c B S}\right)}{P_{u \in i}^{U E}}
$$

The important fact is that the research has modified the formula for received power. This research included the transmitter and receiver gain as well to measure the received power. The reference paper [22] has not included the gain.

\section{Simulation Results and Discussions}

This section of the research presents the empirical measurements based on MATLAB simulation to represent and realize the performance of the small-cell deployed under a macro-cell in a two-tier heterogeneous network. The section thereby includes the simulation results for SINR, throughput, spectral efficiency, and energy efficiency. Table 1 enlists the simulation parameters and values. 
Table 1: Simulation parameters and values

\begin{tabular}{|c|c|}
\hline Measurements and Parameters & Values \\
\hline \multicolumn{2}{|l|}{ 1. SINR Measurement } \\
\hline \multicolumn{2}{|c|}{$\begin{array}{l}\text { 1.1. SINR Measurement (Fixed MBS Power and SBS-MBS Separation } \\
\text { Distance) }\end{array}$} \\
\hline SBS Power & $1-6 \mathrm{~W}$ \\
\hline UE Power & $0.2-2 \mathrm{~W}$ \\
\hline Transmitter Gain (SBS) & $8 \mathrm{~dB}$ \\
\hline Receiver Gain (UE) & $5 \mathrm{~dB}$ \\
\hline SBS-UE (Tx-Rx) Separation & $0-100 \mathrm{~m}$ \\
\hline Interfering SBS Power & $1-6 \mathrm{~W}$ (6 interfering SBSs) \\
\hline MBS Power & $100 \mathrm{~W}$ \\
\hline MBS Antenna Gain & $15 \mathrm{~dB}$ \\
\hline Considered SBS-MBS Separation & $1200 \mathrm{~m}$ \\
\hline \multicolumn{2}{|c|}{ 1.2. SINR Measurement (Fixed SBS Power and SBS-UE Distance) } \\
\hline SBS Power & $6 \mathrm{~W}$ \\
\hline UE Power & $2 \mathrm{~W}$ \\
\hline SBS-UE (Tx-Rx) Separation & $50 \mathrm{~m}$ \\
\hline Interfering SBS Power & $6 \mathrm{~W}$ \\
\hline MBS Power & $10-100 \mathrm{~W}$ \\
\hline MBS Antenna Gain & $15 \mathrm{~dB}$ \\
\hline Considered SBS-MBS Separation & $400-2000 \mathrm{~m}$ \\
\hline \multicolumn{2}{|l|}{ 2. Throughput Measurement } \\
\hline \multicolumn{2}{|c|}{$\begin{array}{l}\text { 2.1. Throughput Measurement (Fixed Transmitter-Receiver Separation } \\
\text { Distance) }\end{array}$} \\
\hline Downlink Bandwidth (SBS) & $1-20 \mathrm{MHz}$ \\
\hline Uplink Bandwidth (UE) & $1-5 \mathrm{MHz}$ \\
\hline SBS-UE (Tx-Rx) Separation & $50 \mathrm{~m}$ \\
\hline SBS Power & $1-6 \mathrm{~W}$ \\
\hline UE Power & $0.2-2 \mathrm{~W}$ \\
\hline \multicolumn{2}{|c|}{ 2.2. Throughput Measurement (Fixed Power of SBS and UE) } \\
\hline Downlink Bandwidth (SBS) & $1-20 \mathrm{MHz}$ \\
\hline Uplink Bandwidth (UE) & $1-5 \mathrm{MHz}$ \\
\hline SBS-UE (Tx-Rx) Separation & $0-100 \mathrm{~m}$ \\
\hline SBS Power & $6 \mathrm{~W}$ \\
\hline UE Power & $2 \mathrm{~W}$ \\
\hline \multicolumn{2}{|c|}{$\begin{array}{l}\text { * In this measurement the parameters of MBS and interfering SBS are } \\
\text { the same as measurement } 1.1 .\end{array}$} \\
\hline \multicolumn{2}{|c|}{$\begin{array}{l}\text { ** For the measurements regarding spectral and power efficiency all the } \\
\text { values for corresponding parameters are the same as measurements } 1 \& \\
2 \text {. }\end{array}$} \\
\hline
\end{tabular}

\subsection{SINR}

The measurement and analysis regarding the SINR for both downlink and uplink are illustrated in this sub-section. Fig. 2 shows the measurements of downlink SINR with respect to transmitter-receiver separation distance and SBS transmission power.

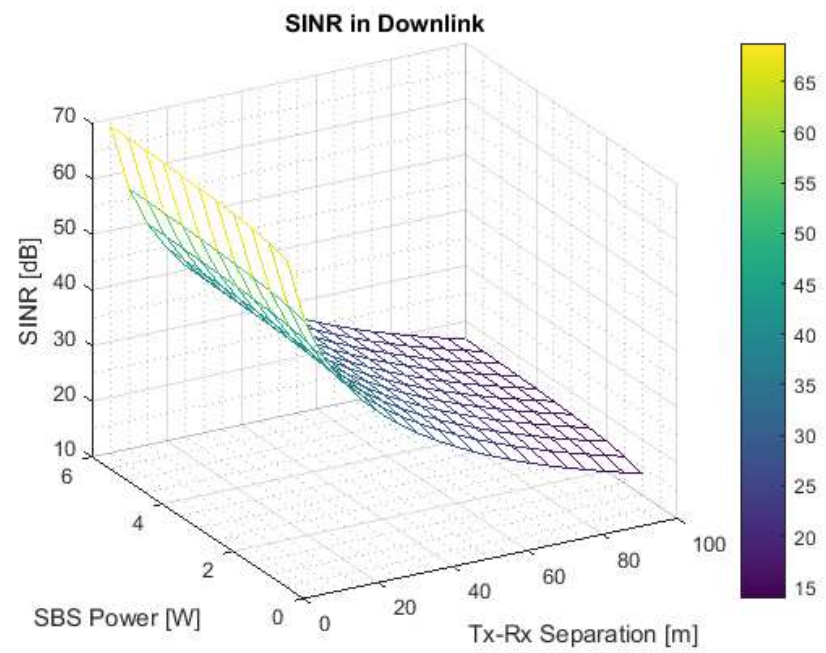

Fig. 2: Downlink SINR

Examining the measurement of downlink SINR the research obtained a maximum of $70 \mathrm{~dB}$ and a minimum of $15 \mathrm{~dB}$ SINR in the downlink. With respect to the considered power, the downlink SINR only varies approximately $4 \mathrm{~dB}$ for maximum to minimum transmission power of the SBS and corresponding transmitter-receiver separation distance.

Fig. 3 shows the measurements of uplink SINR with respect to transmitter-receiver separation distance and UE transmission power.

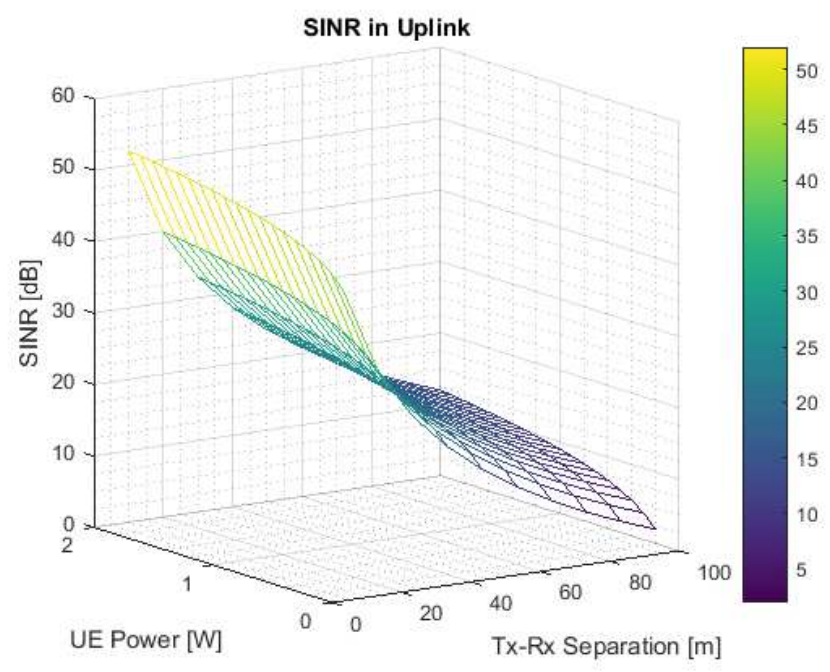

Fig. 3: Uplink SINR

Analyzing the measurement of uplink SINR the research figured a maximum of $52 \mathrm{~dB}$ and a minimum of $2.5 \mathrm{~dB}$ SINR in the uplink. With respect to the considered power of UE, the uplink SINR varies approximately $10 \mathrm{~dB}$ for maximum to minimum power of the UE and corresponding transmitter-receiver separation distance. 
Fig. 4 (a), (b) visualizes the same measurements from a different viewing angle for better realization.

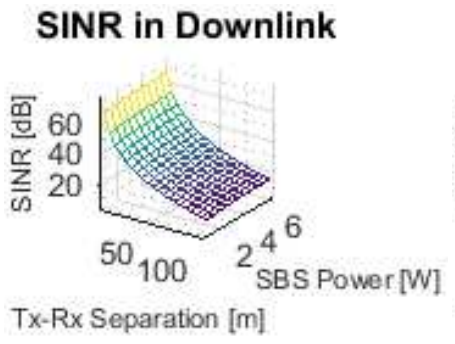

(a)

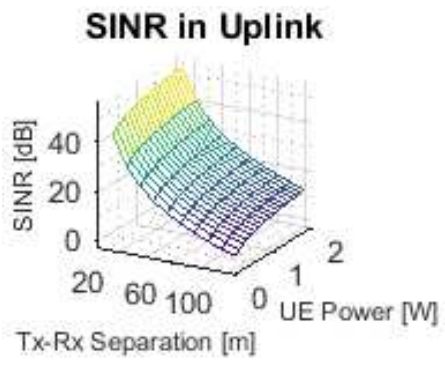

(b)
Fig. 4: (a) Downlink SINR, (b) Uplink SINR

Now, the impact of MBS on the SBS varying parameters such as MBS transmission power, distance is represented by the following simulations. Fig. 5 shows the impact of MBS in the downlink SINR.

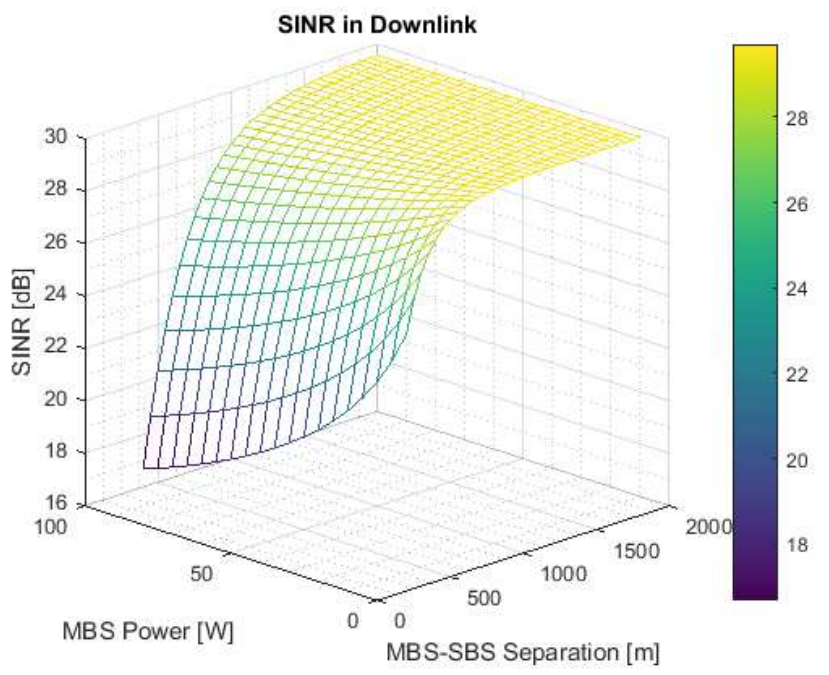

Fig. 5: Downlink SINR

For a shorter separation distance between MBS and SBS, the downlink SINR fluctuates up to $10 \mathrm{~dB}$ for maximum to minimum transmission power of MBS. As the MBS-SBS separation distance increases the fluctuation of SINR (in terms of variation of MBS power) gradually decreases. The research also derived that after a certain distance the impact of MBS on the transmission of SBS almost disappears (or negligible impact). In the case of low transmission power (of MBS) the impact of MBS on the SINR decreases within a shorter transmitter-receiver separation distance compared to the high transmission power of the MBS.

Fig. 6 shows the impact of MBS in the uplink SINR.

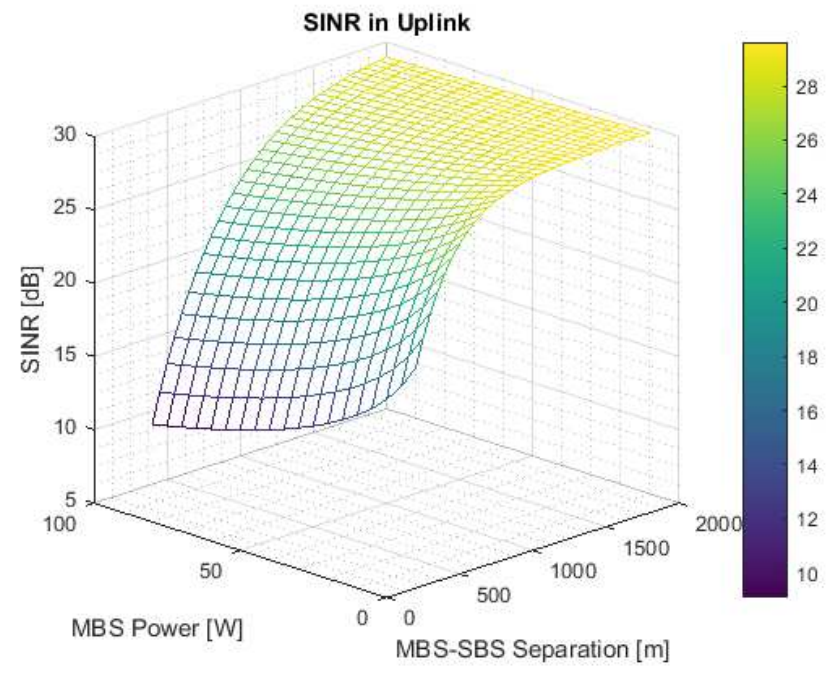

Fig. 6: Uplink SINR

In the case of uplink SINR for a shorter separation distance between MBS and SBS, the uplink SINR fluctuates up to $9 \mathrm{~dB}$ for maximum and minimum transmission power of the MBS. As the MBS-SBS separation distance increases the fluctuation of SINR gradually decreases. After a certain distance, the impact of MBS almost disappears (or becomes negligible). In the case of low transmission power (of MBS) the impact of MBS on the SINR decreases within a shorter transmitter-receiver separation distance compared to the high transmission power of the MBS.

\subsection{Throughput}

This sub-section introduces the measurement of throughput with respect to transmission power (SBS, UE), allocated bandwidth, and transmitter-receiver separation distance. Fig. 7 visualizes the downlink throughput with respect to SBS transmission power and downlink bandwidth for a fixed transmitter-receiver separation distance.

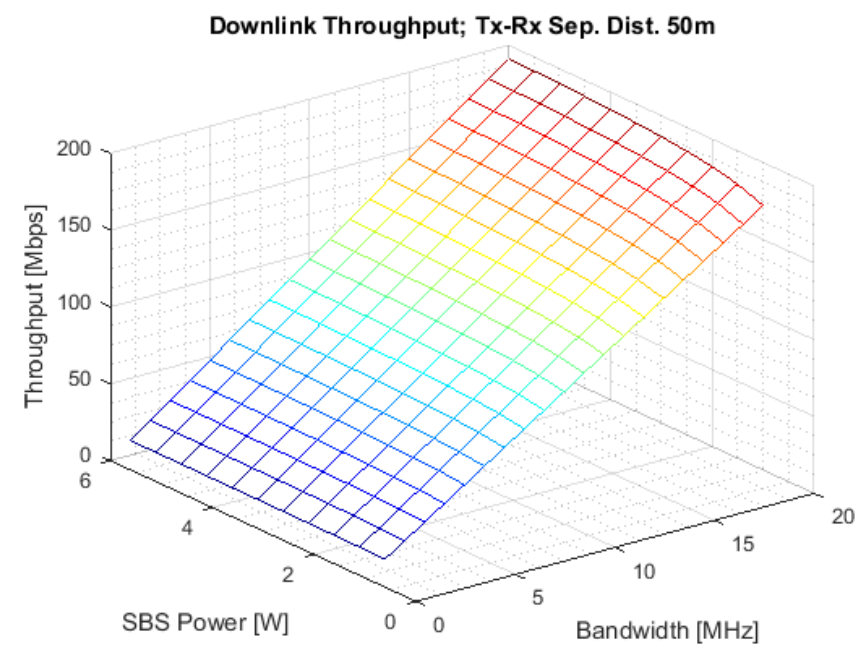

Fig. 7: Downlink throughput 
In the case of downlink throughput for $1 \mathrm{MHz}$ bandwidth and from minimum to maximum transmission power of the SBS the throughput varies up to $1 \mathrm{Mbps}$ (8.603 to $9.568 \mathrm{Mbps}$ ). For maximum bandwidth $(20 \mathrm{MHz})$ and from minimum to maximum transmission power of the SBS the throughput varies up to $19 \mathrm{Mbps}$ (172.1 - $191.4 \mathrm{Mbps}$ ). The measurement is performed considering a separation of $50 \mathrm{~m}$ between transmitter and receiver.

Fig. 8 shows the uplink throughput in terms of allocated SBS transmission power and bandwidth for a fixed transmitterreceiver separation distance.

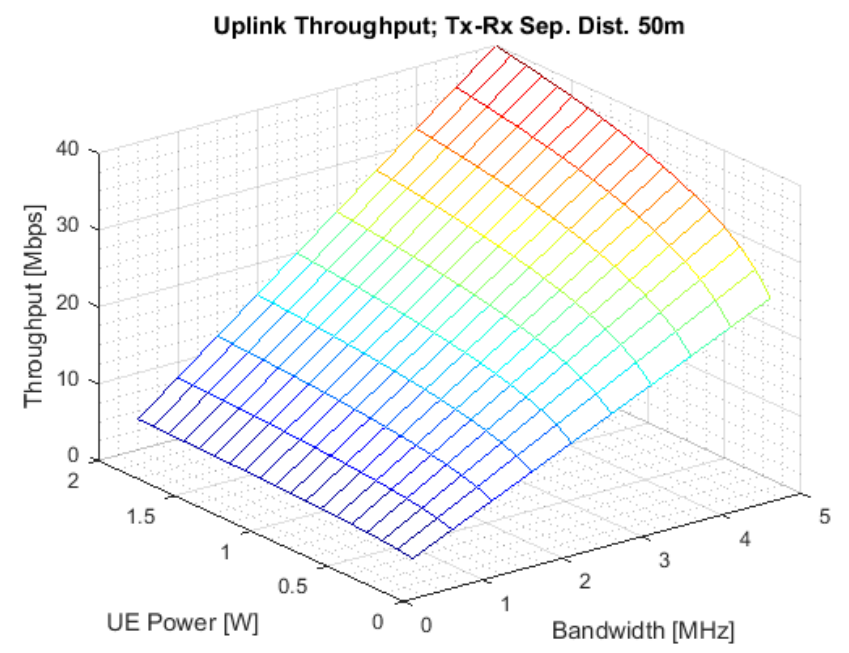

Fig. 8: Uplink throughput

In terms of uplink throughput for minimum $(1 \mathrm{MHz})$ bandwidth and from minimum to maximum transmission power of the UE the throughput varies approx. 1.8 Mbps (2.258 to $3.993 \mathrm{Mbps})$. For maximum bandwidth $(20 \mathrm{MHz})$ and from minimum to maximum transmission power of the UE the throughput varies approx. 16.4 Mbps (23.58 - 39.93 Mbps).

Fig. 9 visualizes the downlink throughput in terms of varied transmitter-receiver separation distance and downlink bandwidth for a fixed SBS transmission power.

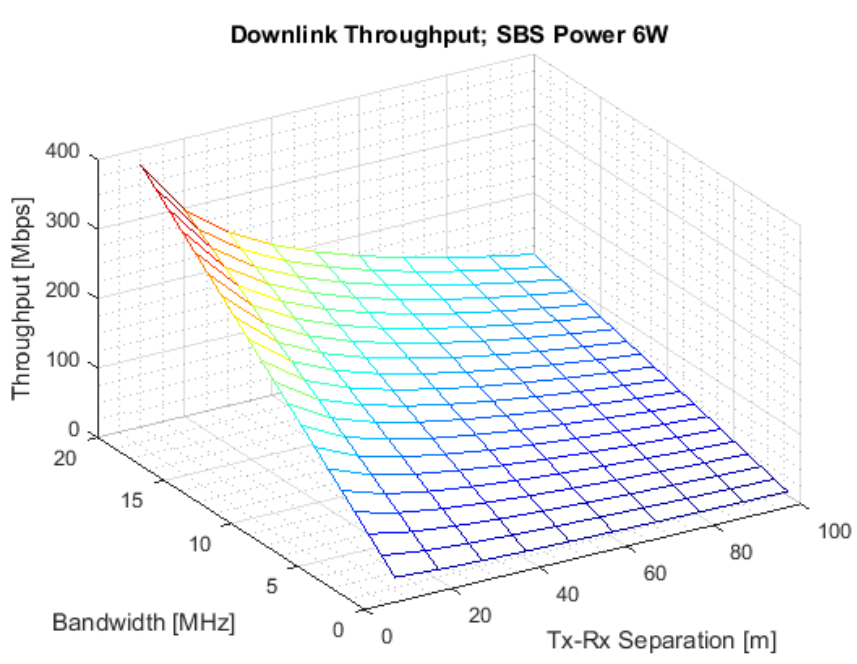

Fig. 9: Downlink throughput

For the shortest transmitter-receiver separation, the network throughput in terms of considered bandwidth highly varies. For $1-20 \mathrm{MHz}$ bandwidth, the throughput varies from $18.85-$ 377.1 Mbps (approx. $358 \mathrm{Mbps)}$ ) for the shortest transmitter and receiver separation distance. But with the increment of transmitter-receiver separation distance, this variation of throughput gradually decreases. In the case of the highest transmitter and receiver separation distance, the throughput varies from 5.596 - 111.9 Mbps (approx. $107 \mathrm{Mbps)}$ ) with respect to bandwidth considered.

Fig. 10 illustrates the measurement of uplink throughput in terms of varied transmitter-receiver separation distance and uplink bandwidth for a fixed SBS transmission power.

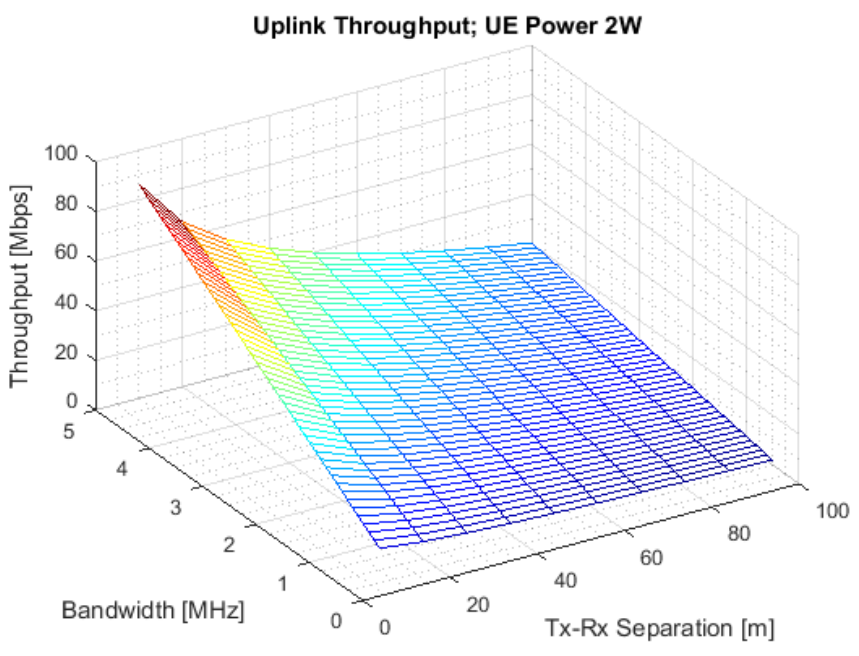

Fig. 10: Uplink throughput

In the case of uplink, the network throughput for the shortest transmitter-receiver separation distance varies highly compared to the maximum transmitter-receiver separation distance. With respect to the allocated bandwidth, the throughput varies from 
8.634 - 86.34 Mbps (approx. 78 Mbps) for the shortest transmitter and receiver separation distance. But with the increment of transmitter-receiver separation distance, this variation of throughput gradually decreases. In the case of the highest transmitter and receiver separation distance, the throughput varies from 2.035 - 20.35 Mbps (approx. $18 \mathrm{Mbps}$ ) with respect to bandwidth considered.

\subsection{Spectral Efficiency}

In this sub-section, the measurements regarding the spectral efficiency for both downlink and uplink are represented. Fig. 11 shows the downlink spectral efficiency.

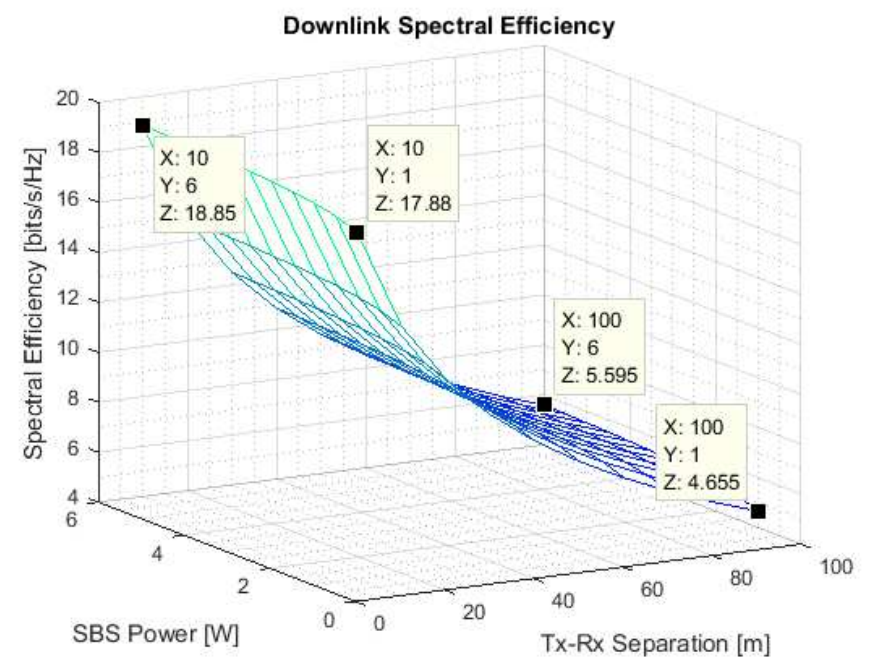

Fig. 11: Downlink spectral efficiency

Fig. 12 represents the uplink spectral efficiency.

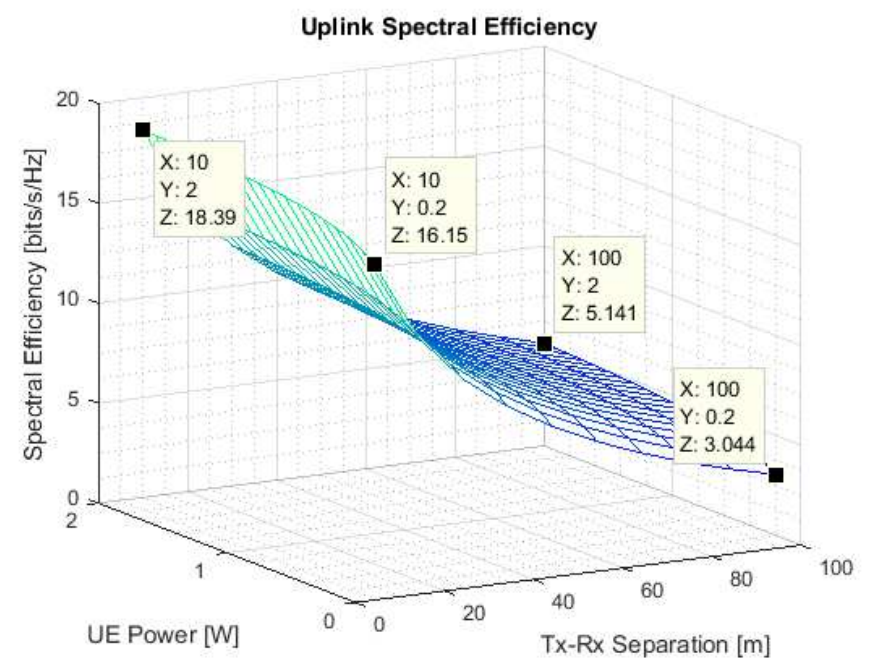

Fig. 12: Downlink spectral efficiency

Fig. 13 (a), (b) visualizes the same measurements from a different viewing angle for better realization.

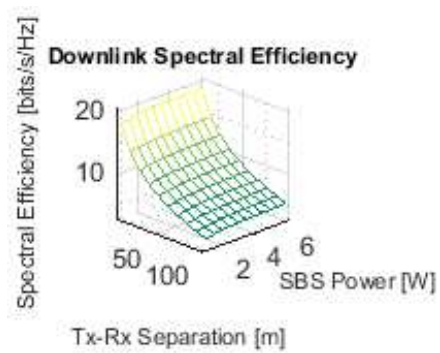

(a)

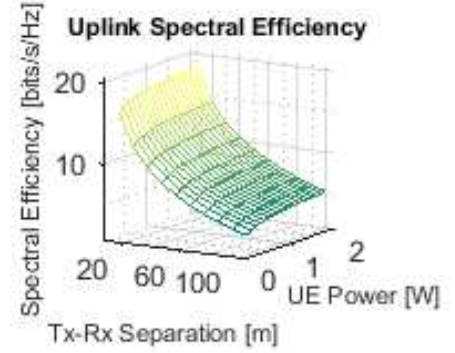

(b)
Fig. 13: (a) Downlink spectral efficiency, (b) Uplink spectral efficiency

The research derived that the spectral efficiency for both downlink and uplink is analogous and there is not much difference in the characteristics. But in the case of uplink, the spectral efficiency varies a bit higher than the downlink with respect to considered transmission power and transmitterreceiver separation distance.

\subsection{Power Efficiency}

This sub-section represents the simulations regarding the downlink and uplink power efficiency. First of all, the research performed the measurements of downlink and uplink power efficiency varying the SBS and UE power, allocated bandwidth, and keeping the transmitter-receiver separation distance fixed. Fig. 14 shows the downlink power efficiency.

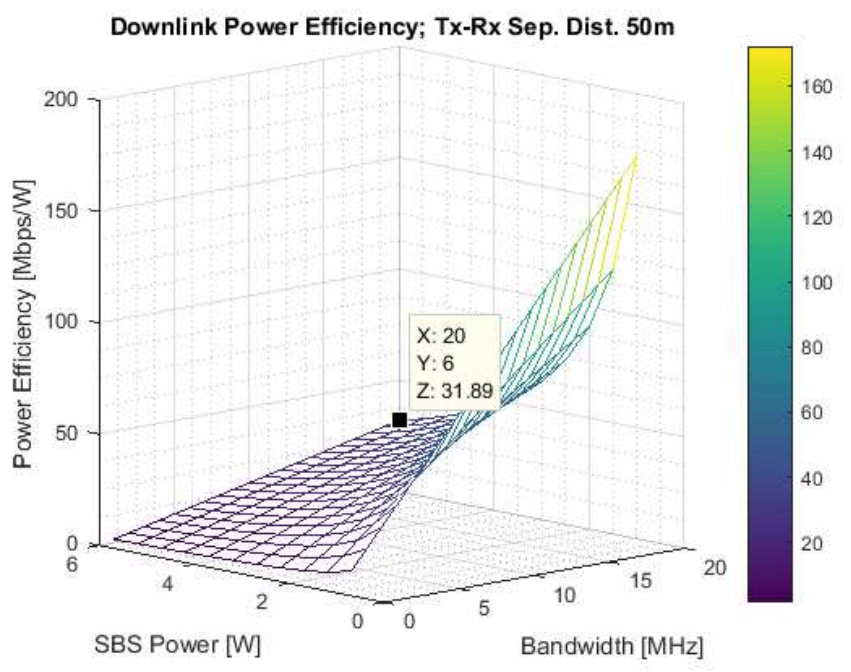

Fig. 14: Downlink power efficiency

Examining the above simulation the research figured out that, with respect to the allocated bandwidth $(1-20 \mathrm{MHz})$ for the lowest transmission power of the SBS the power efficiency varies highly approximately $163.5 \mathrm{Mbps} / \mathrm{W}(8.603-172.1$ $\mathrm{Mbps} / \mathrm{W})$. With the increment of the SBS, power the variation of power efficiency gradually decreases. For the maximum transmission power of the SBS, the variation of power efficiency for the minimum to maximum allocated bandwidth is approximately $30 \mathrm{Mbps} / \mathrm{W}(1.595-31.89 \mathrm{Mbps} / \mathrm{W})$. 
Fig. 15 visualizes the downlink power efficiency.

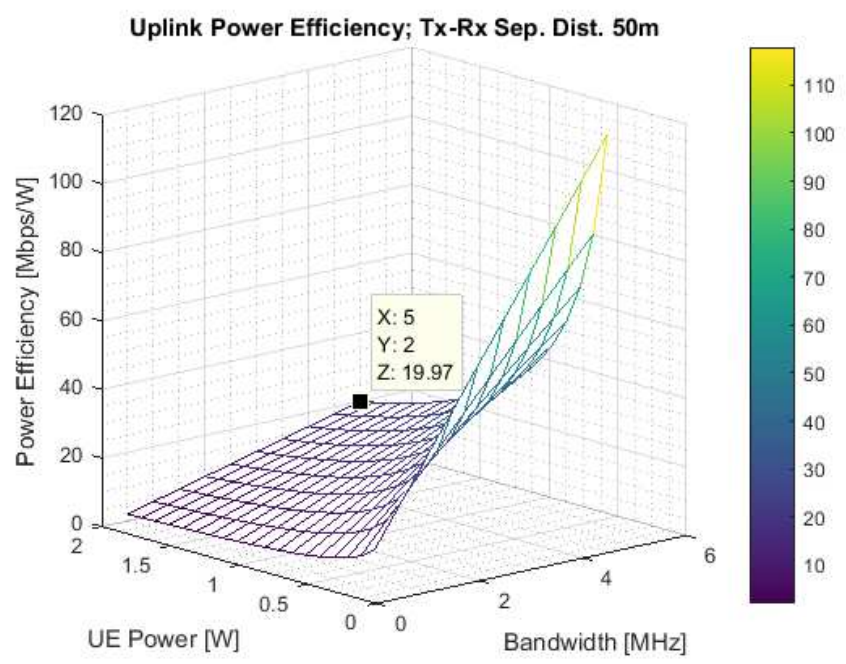

Fig. 15: Uplink power efficiency

In the case of uplink, with respect to the allocated bandwidth (1 $-20 \mathrm{MHz}$ ) for the lowest transmission power of the UE, the power efficiency varies highly approximately $106.11 \mathrm{Mbps} / \mathrm{W}$ $(11.79-117.9 \mathrm{Mbps} / \mathrm{W})$. With the increment of the UE power, the high variation of power efficiency gradually decreases. For the maximum transmission power of the UE (considered for this research) the variation of power efficiency for the minimum to maximum allocated bandwidth is approximately $18 \mathrm{Mbps} / \mathrm{W}$ (1.997 - 19.97 Mbps/W).

This time, the research performed the measurements of downlink and uplink power efficiency varying the transmitter-receiver separation distance, allocated bandwidth, and keeping the SBS and UE transmission power fixed. Fig. 16 shows the downlink power efficiency.

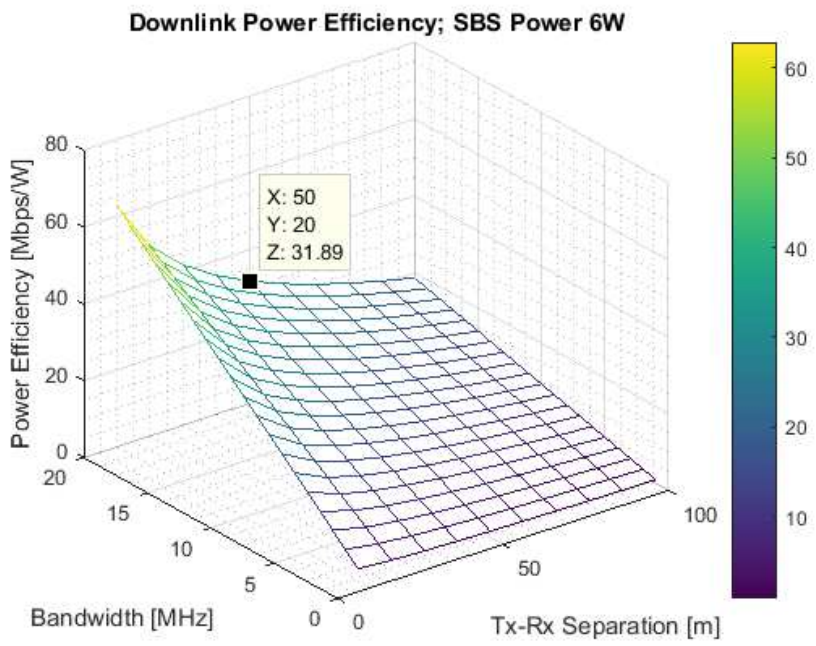

Fig. 16: Downlink power efficiency
With respect to the increment of transmitter-receiver separation distance $(0-100 \mathrm{~m})$, the power efficiency for the lowest bandwidth varies a little approximately $2 \mathrm{Mbps} / \mathrm{W}(0.9327$ $3.142 \mathrm{Mbps} / \mathrm{W}$ ) compared to the maximum allocated bandwidth. For the maximum allocated bandwidth, the variation of power efficiency with respect to the distance $(0-100 \mathrm{~m})$ is comparatively high approximately $44 \mathrm{Mbps} / \mathrm{W}$ (18.65 - 62.85 $\mathrm{Mbps} / \mathrm{W}$ ). Observing these phenomena it can be stated that with the increment of bandwidth the amount of the variation of power efficiency gets increased with respect to the transmitter-receiver separation distance.

Fig. 17 shows the uplink power efficiency.

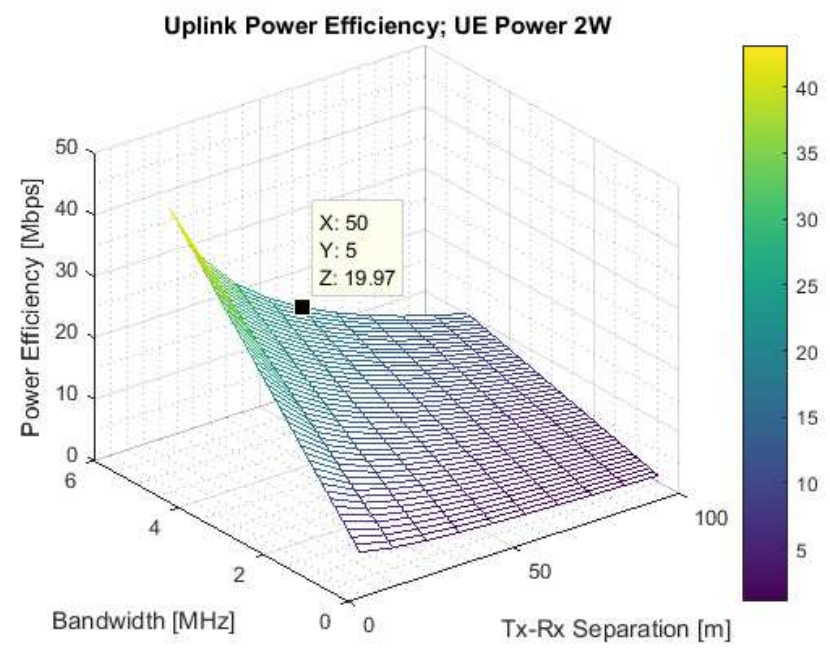

Fig. 17: Uplink power efficiency

In the case of uplink, the variation of power efficiency is analogous compared to the downlink. With respect to the increment of transmitter-receiver separation distance $(0-100$ $\mathrm{m})$, the power efficiency for the minimum bandwidth varies a little approximately $3.3 \mathrm{Mbps} / \mathrm{W}(1.017-4.317 \mathrm{Mbps} / \mathrm{W})$ compared to the maximum allocated bandwidth. For the maximum allocated bandwidth, the variation of power efficiency with respect to the distance $(0-100 \mathrm{~m})$ is comparatively high approximately $33 \mathrm{Mbps} / \mathrm{W}(10.17-43.17 \mathrm{Mbps} / \mathrm{W})$.

\section{Research Outcomes and Future Directions}

\subsection{Notable Outcomes of the Research}

This sub-section outlines the notable outcomes and findings derived from the research. These are,

SINR: The SINR for both downlink and uplink highly varies with the variation of transmitter-receiver separation distance. But it varies in small-scale with the variation of transmission power. In the case of low transmission power (of MBS) the impact of MBS on the SINR decreases within a shorter transmitter-receiver separation distance compared to the high transmission power of the MBS. 
Throughput: For lower bandwidth, the variation of throughput with respect to the variation of transmission power is comparatively lower (almost negligible) than the higher value of allocated bandwidth. For the shortest transmitter-receiver separation, the network throughput highly varies with respect to the variation of allocated bandwidth (minimum to maximum). With the increment of transmitter-receiver separation distance, this variation of throughput gradually decreases and for the longest separation distance, the variation of throughput is less compared to the shortest separation (with respect to the variation of bandwidth).

Spectral Efficiency: The spectral efficiency for both downlink and uplink is analogous according to the findings of this research. But in the case of uplink, the spectral efficiency varies a bit higher than the downlink with respect to considered transmission power and transmitter-receiver separation distance.

Power Efficiency: With respect to the variation of allocated bandwidth for the lowest transmission power the power efficiency varies highly. With the increment of the transmission power the variation of power efficiency gradually decreases (for the variation of allocated bandwidth). For the maximum transmission power, the variation of power efficiency with respect to the variation of allocated bandwidth is low. The power efficiency varies on a small-scale with respect to the variation of transmitter-receiver separation distance in terms of low bandwidth. But the power efficiency varies highly with respect to the transmitter-receiver separation distance in terms of high bandwidth.

\subsection{Research Directions}

This segment portrays several research directions on which advanced research and study can be carried out to lead the efficient implementation of small-cells in a heterogeneous network. These are,

- Further assessments may be carried out considering increased transmission power, bandwidth, SBS-UE separation distance, SBS-MBS separation distance, etc.

- Measurements can be performed in further researches considering channel gain (considering formulas mentioned in this research).

- Measurements can be performed considering a 3D deployment scenario.

- Algorithms can be developed considering the formulas of this research for optimal resource allocation.

\section{Conclusion}

The research has characterized the deployment of small-cell under a two-tier heterogeneous network considering the impact of a macro-cell in terms of SINR, throughput, spectral efficiency, and power efficiency. The notable thing of the research is that it has modified the received power formula by including the transmitter and receiver gain. The researchers then measured the SINR, throughput, spectral efficiency, and power efficiency by simulation approaches and briefed the notable findings from those simulations. The paper also included some further research and enhancement matters for future works. The research pretends to be assistive to enthusiasts, academicians, researchers, and scholars currently engaged in research for the efficient deployment of small-cells in a 5G heterogeneous network.

\section{References}

[1] M. H. Alsharif, R. Nordin, "Evolution towards fifth generation (5G) wireless networks: Current trends and challenges in the deployment of millimetre wave, massive MIMO, and small cells," in Telecommunications Systems, vol. 64, pp. 617-637, April 2017. doi: 10.1007/s11235-016-0195-x.

[2] R. Chávez-Santiago et al., "5G: The Convergence of Wireless Communications," in Wireless Personal Communications, vol. 83, pp. 1617-1642, March 2015. doi: 10.1007/s11277-015-2467-2.

[3] M. Hawasli, S. A. Çolak et al., "Toward green 5G heterogeneous small-cell networks: power optimization using load balancing technique," in AEU - International Journal of Electronics and Communications, vol. 82, pp. 474-485, December 2017. doi: 10.1016/j.aeue.2017.09.012.

[4] A. S. Bhosle, "Emerging trends in small-cell technology," 2017 IEEE International Conference on Electrical, Instrumentation and Communication Engineering (ICEICE), Karur, India, 2017, pp. 14, doi: 10.1109/ICEICE.2017.8191847.

[5] W. Yang, J. Zhang and J. Zhang, "On Evaluation of Indoor to Outdoor Communications Using Neighbourhood Small Cells," in IEEE Transactions on Vehicular Technology, vol. 69, no. 7, pp. 8045-8050, July 2020, doi: 10.1109/TVT.2020.2993401.

[6] M. H. Alsharif, R. Nordin, M. M. Shakir et al., "Small Cells Integration with the Macro-Cell Under LTE Cellular Networks and Potential Extension for 5G" in Journal of Electrical Engineering \& Technology, vol. 14, pp. 2455-2465, April 2019. doi: 10.1007/s42835-019-00173-2.

[7] I. P. Chochliouros et al., "Using small cells for enhancing 5G network facilities," 2017 IEEE Conference on Network Function Virtualization and Software Defined Networks (NFV-SDN), Berlin, Germany, 2017, pp. 264-269, doi: 10.1109/NFVSDN.2017.8169871.

[8] M. M. Fadoul, "Modeling multi-tier heterogeneous small cell networks: rate and coverage performance," in Telecommunications Systems, vol. 75, pp. 369-382, July 2020. doi: 10.1007/s11235020-00680-y.

[9] D. Abonyi and D. Rigelsford, "A System for Optimizing SmallCell Deployment in 2-Tier HetNets," 2018 IEEE 23rd International Workshop on Computer Aided Modeling and Design of Communication Links and Networks (CAMAD), Barcelona, Spain, 2018, pp. 1-6, doi: 10.1109/CAMAD.2018.8514981.

[10] Y. A. Sambo et al., "K-tier heterogeneous small-cell networks: Towards balancing the spectrum usage and power consumption with aggressive frequency reuse," 2013 First International Black Sea Conference on Communications and Networking (BlackSeaCom), Batumi, Georgia, 2013, pp. 132-136, doi: 10.1109/BlackSeaCom.2013.6623396.

[11] A. R. Ramos, B. C. Silva, M. S. Lourenço, E. B. Teixeira and F. J. Velez, "Mapping between Average SINR and Supported Throughput in 5G New Radio Small Cell Networks," 2019 22nd International Symposium on Wireless Personal Multimedia Communications (WPMC), Lisbon, Portugal, 2019, pp. 1-6, doi: 10.1109/WPMC48795.2019.9096179. 
[12] T. Elkourdi and O. Simeone, "Femtocell as a Relay: An Outage Analysis," in IEEE Transactions on Wireless Communications, vol. 10, no. 12, pp. 4204-4213, December 2011, doi: 10.1109/TWC.2011.100611.102046.

[13] D. Wu, Jun Gu, Xinyu Gu, Shiwen Nie, Ming Zhao and Lin Zhang, "User average data rate analysis in future dense small cells," 2015 IEEE 16th International Conference on Communication Technology (ICCT), Hangzhou, China, 2015, pp. 481-486, doi: 10.1109/ICCT.2015.7399883.

[14] H. Shimodaira et al., "Optimization of picocell locations and its parameters in heterogeneous networks with hotspots," 2012 IEEE 23rd International Symposium on Personal, Indoor and Mobile Radio Communications - (PIMRC), Sydney, NSW, Australia, 2012, pp. 124-129, doi: 10.1109/PIMRC.2012.6362538.

[15] A. Pratap, R. Misra and S. K. Das, "Resource Allocation to Maximize Fairness and Minimize Interference for Maximum Spectrum Reuse in 5G Cellular Networks," 2018 IEEE 19th International Symposium on "A World of Wireless, Mobile and Multimedia Networks" (WoWMoM), Chania, 2018, pp. 1-9, doi: 10.1109/WoWMoM.2018.8449760.

[16] Y. Pak, K. Min and S. Choi, "Performance evaluation of various small-cell deployment scenarios in small-cell networks," The 18th IEEE International Symposium on Consumer Electronics (ISCE 2014), Jeju, Korea (South), 2014, pp. 1-2, doi: 10.1109/ISCE.2014.6884453.

[17] A. A. Al Haija and C. Tellambura, "Small-Macro Cell Cooperation for HetNet Uplink Transmission: Spectral Efficiency and Reliability Analyses," in IEEE Journal on Selected Areas in Communications, vol. 35, no. 1, pp. 118-135, Jan. 2017, doi: 10.1109/JSAC.2016.2633039.

[18] W. Guo, S. Wang, X. Chu, J. Zhang, J. Chen and H. Song, "Automated small-cell deployment for heterogeneous cellular networks," in IEEE Communications Magazine, vol. 51, no. 5, pp. 46-53, May 2013, doi: 10.1109/MCOM.2013.6515046.

[19] A. Maltsev et al., "Performance evaluation of interference mitigation techniques in the overlaying MmWave small cell network," 2015 IEEE Conference on Standards for Communications and Networking (CSCN), Tokyo, Japan, 2015, pp. 130-136, doi: 10.1109/CSCN.2015.7390433.

[20] S. M. Ahsan Kazmi et al., "Resource management in dense heterogeneous networks," 2015 17th Asia-Pacific Network Operations and Management Symposium (APNOMS), Busan, Korea (South), 2015, pp. 440-443, doi: 10.1109/APNOMS.2015.7275383.

[21] H. Pervaiz, L. Musavian and Q. Ni, "Area energy and area spectrum efficiency trade-off in 5G heterogeneous networks," 2015 IEEE International Conference on Communication Workshop (ICCW), London, UK, 2015, pp. 1178-1183, doi: 10.1109/ICCW.2015.7247337.

[22] T. Mir, L. Dai, Y. Yang, W. Shen and B. Wang, "Optimal FemtoCell Density for Maximizing Throughput in 5G Heterogeneous Networks under Outage Constraints," 2017 IEEE 86th Vehicular Technology Conference (VTC-Fall), Toronto, ON, Canada, 2017, pp. 1-5, doi: 10.1109/VTCFall.2017.8288059. 